\title{
Indications, Interpretation and Clinical Consequences of Tuberculin Skin Tests in Resource Limited Settings
}

\author{
Fitzgerald A. Gopie ${ }^{1,2^{*}}$ (D) Aabidien Hassankhan ${ }^{3}$ (D), Wilco C. W. R. Zijlmans ${ }^{1,4}$ (D), \\ Stephen G. S. Vreden 5 (1) \\ ${ }^{1}$ Faculty of Medical Sciences, Anton de Kom University of Suriname, Paramaribo, Suriname \\ ${ }^{2}$ Academic Hospital Paramaribo, Paramaribo, Suriname \\ ${ }^{3}$ MoleMann Mental Health Suriname, Paramaribo, Suriname \\ ${ }^{4}$ Department of Paediatrics, Diakonessenhuis Hospital, Paramaribo, Suriname \\ ${ }^{5}$ Department of Internal Medicine, Academic Hospital Paramaribo, Paramaribo, Suriname \\ Email:^fitzgeraldgopie@gmail.com,^fgopie@azp.sr, adienkhan@live.com,cwrzijlmans@gmail.com, stephenvreden@yahoo.com
}

How to cite this paper: Gopie, F.A., Hassankhan, A., Zijlmans, W.C.W.R. and Vreden, S.G.S. (2021) Indications, Interpretation and Clinical Consequences of Tuberculin Skin Tests in Resource Limited Settings. Journal of Tuberculosis Research, 9, 172-183. https://doi.org/10.4236/jtr.2021.93016

Received: July 6, 2021

Accepted: August 13, 2021

Published: August 16, 2021

Copyright $\odot 2021$ by author(s) and Scientific Research Publishing Inc. This work is licensed under the Creative Commons Attribution International License (CC BY 4.0).

http://creativecommons.org/licenses/by/4.0/

\begin{abstract}
Objective: to evaluate the policy of TST testing in Suriname. As there is no gold standard to diagnose latent tuberculosis infection (LTBI), the tuberculin skin test (TST) is used to diagnose LTBI. However, internationally, the cut-off values of the TST are not uniform and depend on local tuberculosis (TB) epidemiology and guidelines for test initiation. In Suriname, where currently several indications exist for TSTs, cut-off values are set at $5 \mathrm{~mm}$ or $10 \mathrm{~mm}$, depending on the age and/or medical history of the patient. LTBI classification is performed by pulmonologists primarily based on the American Thoracic Society targeted TB testing guidelines. Method: retrospective analysis of outpatient TST data between 2011 and 2019 from Suriname's sole pulmonary medicine clinic. Result: 1373 patients were evaluated. 590 patients were from the screening group of whom 253 had a positive TST result, 46 of whom were classified as LTBI. In the contact tracing group of 649 patients, 616 had a positive TST, 352 of whom were classified as LTBI. In the medical condition group of 134 patients, 96 had a positive TST, 38 of whom were classified as LTBI. Eventually, positive TST results were found for 965 tested patients: 436 patients were classified as LTBI and 529 non-LTBI patients were not prescribed chemoprophylaxis. None of the non-LTBI TST-positive patients were diagnosed with active TB, including 174 patients with a TST result of $15 \mathrm{~mm}$ or greater and in need of IPT, but not prescribed by judgement of the pulmonologist or because of loss to follow-up. Conclusion: the overrepresentation of positive TST results in Suriname is attributable to stringent cut-off values,
\end{abstract}


especially among patients who do not disclose TB risk factors. In our opinion the TST cut-off value for such patients in Suriname and other similar settings could be set at $15 \mathrm{~mm}$. We also promote that for all patients with a TST result of $15 \mathrm{~mm}$ or greater, offering IPT should be considered (after excluding active TB).

\section{Keywords}

Tuberculin Skin Test, Cut-Off Values, Guidelines, Revision

\section{Introduction}

Infection of humans by tuberculosis (TB) bacilli can either result in no disease symptoms and spontaneous healing, active disease (with pulmonary TB being the most common and infective form), or latent TB infection (LTBI). The latter, non-infective form [1] [2], is the major source of new active TB cases [3] [4] [5] [6]. In 2014, some 1.7 billion people were estimated to have LTBI, equivalent to $23 \%$ of the world population [4]. LTBI is defined by WHO as "a state of persistent immune response to stimulation by Mycobacterium tuberculosis antigens with no evidence of clinically manifest active TB". The lifetime risk of LTBI reactivation in healthy individuals ranges from $5 \%$ to $15 \%$ but may increase depending on concomitant risk factors and comorbidity, eventually resulting in the potential development of active TB. As such, people living with HIV (PLHIV) have about a 100 -fold increased risk of developing active TB [7].

As there is no gold standard to diagnose LTBI [3] [5] [7], TB contact screening, Interferon Gamma Release Assays (IGRAs), and tuberculin skin tests (TSTs) are used in clinical practice to evaluate LTBI. The IGRA has a better predictive ability but is expensive compared to the TST, which in turn has the disadvantage of requiring multiple visits to the test facility [8]. According to criteria of the American Thoracic Society (ATS), skin indurations of $5 \mathrm{~mm}$ or $10 \mathrm{~mm}$ are classified as a positive TST result depending on the patient's age, HIV status, TB exposure, chest X-ray findings, comorbidities, injection drug use, and occupation. A TST result of $15 \mathrm{~mm}$ or greater is considered positive in persons with no risk factors for TB [9] [10] [11].

Suriname, a low- and middle-income country in the South American tropics, can be considered a low TB incidence country (defined as a TB incidence rate of less than 100 per 100,000) [7], with an estimated TB incidence of 24 per 100,000 in 2000, 46 per 100,000 in 2010, and 29 per 100,000 in 2019 [12]. In 2012, the National Tuberculosis Program (NTP) launched the first national TB guidelines on behalf of the Ministry of Health. Per these guidelines, the cut-off values for TSTs were set at $5 \mathrm{~mm}$ for PLHIV and children under 5 years of age and $10 \mathrm{~mm}$ for all other persons [13].

For decades, Bacillus Calmette-Guerin (BCG) vaccinations have not been administered in Suriname. As it turns out, the BCG vaccination program carried out 
from 1955 to 1956 among 56,000 of Suriname's inhabitants was the last mass vaccination program. Nonspecific tuberculin sensitivity in Suriname seems to be frequent, as reported by Bleiker and van Erpecum from 1962 to 1965 and by van Weissenbruch in 1979.

Between 1966 and 1971, screening for TB using chest X-ray among 60,301 people resulted in signs of TB for only 60 people. Also, from 1965 to 1974, a tuberculin survey among 156,000 people revealed only 88 positive cases [14]. The population of Suriname was 379,607 in 1970 [15].

Nontuberculous Mycobacteria (NTM) are commonly present in Suriname [16]. Between 2007 and 2011, growth of NTM was detected in sputum cultures from patients suspected of TB, varying from $30.1 \%$ in 2009 to $47.7 \%$ in 2007 , with a yearly average of $39.2 \%$. M. tuberculosis growth during this time was noted in $50.6 \%$ of the sputum cultures of patients treated for TB (data from the national tuberculosis laboratory of the Central Laboratory, Bureau of Public Health).

The WHO encourages countries to develop and implement national TB guidelines at their own discretion [7]. As such, all patients with TB in Suriname should be registered with the NTP, a government workforce that is responsible for TB contact tracing and public health measures regarding TB. Since IGRAs are not available in Suriname, the TST is used to identify LTBI. Other than for TB contact tracing, in Suriname the TST is sometimes required for a work permit or for admission to a nursing home. In addition, countries like the USA and Belgium require a negative TST result or LTBI treatment from Surinamese nationals for the purposes of emigration or study.

Having utilized the TST cut-off values of $5 \mathrm{~mm}$ and $10 \mathrm{~mm}$ for many years, we had the impression of over designation of positive TST results, as a consequence of the NTP guidelines. As such we were curious to evaluate how the TST results, of TB contact tracing patients, screening patients, and patients with a medical condition other than $\mathrm{TB}$, were dealt with in clinical practice and if adjustments needed to be made to the TST policy of Suriname. Our investigative parameters were a) the reason a TST was performed; b) the numerical value and designation of the TST; and c) the policy pursued by the treating physician and eventually the pulmonologist.

\section{Method}

\subsection{TST Testing Algorithm and Patient Workup}

Indication: to evaluate tuberculosis exposure, the TST can be ordered by any physician practicing in Suriname, which is performed by the NTP with purified protein derivative (PPD RT23/SSI Copenhagen Denmark) (Figure 1). NTP personnel measure the skin induration in $\mathrm{mm}$ and designate the TST result as negative or positive, according to the NTP guidelines. Tested persons with a positive TST result are advised to consult a medical specialist for additional evaluation, namely a pulmonologist for adults and a pediatrician for children.

Interpretation: for the classification of a positive TST test result a detailed anam- 

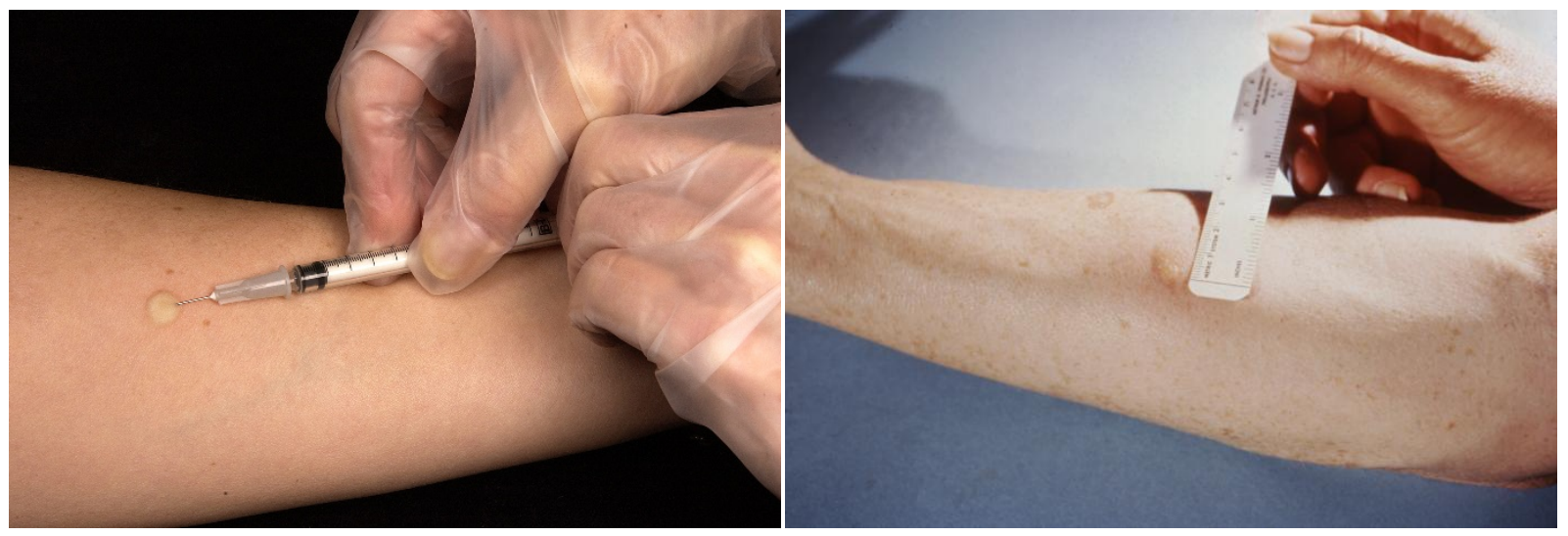

Source. Centers for Disease Control and Prevention, Public Health Image Library, ID\#: 6806 (left), 3752 (right).

Figure 1. Placement and measurement of the tuberculin skin test.

nesis is taken at the pulmonology clinic and a chest X-ray is performed, which is evaluated for pulmonary lesions like consolidation, broadened hila, granulomas and miliary TB [17]. Subsequently the pulmonologist categorizes the TST as done in case of 1) screening (for a work permit, admittance to a nursing home, study abroad, or emigration); 2) TB contact tracing; or 3) an additional test to rule out LTBI in patients with medical conditions like chronic cough, weight loss, fatigue, hemoptysis, and ocular disease. Patients referred for TB contact tracing and medical conditions also have their sputum examined for acid fast bacilli (AFB) with Ziehl-Neelsen (ZN) staining and, from 2012 onwards, with the Xpert MTB/RIF (Cepheid Sunnyvale). After this work up it is decided by the pulmonologist, having ruled out active TB and considering the anamnesis and chest $\mathrm{X}$-ray result, if a patient with a positive TST result is at risk for developing TB and as such needs to be classified as LTBI.

Clinical consequence: no follow-up is conducted by the pulmonologist if a person has a negative TST result and is without an anamnesis or signs of TB. In the TB contact tracing group and the medical condition group, patients classified as LTBI [9] by the pulmonologist are offered isoniazid preventative treatment (IPT), whereas patients with a positive TST but not classified as LTBI are assigned to a "wait and see, no IPT" policy for 2 years-as this is the most likely time for TB reactivation to occur [5] - except in the presence of comorbidities such as diabetes mellitus (DM), HIV, cancer, and chronic renal failure. No follow-up or "wait and see, no IPT" policy is applied to screening group patients who have a positive TST result (as designated by the NTP) but who were not classified as LTBI by the pulmonologist.

Patients classified as LTBI are offered 6 months of IPT and are monitored by pulmonologist for adverse reactions, liver function tests-aspartate aminotransferase (AST) and alanine transaminase (ALT) - and treatment adherence. IPT is completed after 6 months of isoniazid intake, during which the patient should have no signs of active TB. If an LTBI patient declines IPT or does not complete IPT, follow-up evaluations are offered for a period of 2 years [5]. 


\subsection{Study Protocol}

We compiled outpatient data and conducted a study regarding the TST results of patients-who had a TST done at the NTP between January $1^{\text {st }}, 2011$ and December $31^{\text {st }}, 2019$ and were referred to Suriname's sole pulmonary medicine clinic at the Academic Hospital Paramaribo (children were mostly referred to the pediatrician). Follow-up data of these patients were collected until December 31 32020. Excluded from analysis were patients who had been treated for TB or presented with active TB and persons who had no numerical value for their TST result. The extracted data were anonymized and categorized into three TST indication groups (i.e., the screening, TB contact tracing, and medical condition groups), and included: sex, age, TST indication and TST result, co-morbidities known to be risk factors for TB [7], and the interventions undertaken by the pulmonologist.

\subsection{Statistics}

Statistical analyses included the calculation of follow-up durations using the midyear date of the year in which the TST was performed (the exact dates on which TSTs were carried out were unavailable) and the end date of the study period. Differences between TST groups-which are based on the reason for the test: screening, TB contact tracing, and medical condition-on selected variables were analyzed with chi-square tests for categorical variables. Statistical analyses were performed using SPSS version 20.0 (computer software; IBM Corp., 2011). For significance testing, all alpha levels were set at 0.05 .

\subsection{Ethics}

Our study was performed with anonymized patient data from our own clinic and was approved by the Ministry of Health.

\section{Results}

During the study period, 1384 patients with a TST result were referred. Excluded from analysis were 11 patients who had no numerical TST result. Data from 1373 patients were analyzed. Mean age at the time of referral was 37.3 years; the average follow-up time for all positive patients was 4.3 years. Our study cohort included 96 children, of whom 11 were under the age of 5 years; 85 were between 5 and 15 years.

Based on the NTP cut-off values, 408 patients (29.7\%) had a negative TST result (including 24 patients from the TB contact tracing group with a TST result between $5 \mathrm{~mm}$ and under $10 \mathrm{~mm}$; see Table 1 and Figure 2). 965 patients (70.3\%; including a 4-year-old girl with a TST result of $8 \mathrm{~mm}$ ) had a positive TST result (of $10 \mathrm{~mm}$ or greater unless otherwise noted), including 9 patients who developed skin blisters of up to $55 \mathrm{~mm}$ after PPD injection. In none of the patients with a positive TST result was active TB diagnosed by the pulmonologist. Finally, 436 patients (45.2\%) with a positive TST result were classified as LTBI by the pulmonologist. 
Table 1. Patient classification with selected details.

\begin{tabular}{ccc}
\hline Classification & $\mathbf{n}$ & $\%$ \\
\hline Total & 1373 & $100 \%$ \\
TST negative & 408 & $29.7 \%$ \\
TST positive & 965 & $70.3 \%$ \\
No LTBI & 529 & $54.8 \%$ \\
TST under 15 mm & 355 & $67.1 \%$ \\
TST $\geq 15$ mm & 174 & $32.9 \%$ \\
LTBI & 436 & $45.2 \%$ \\
No IPT administered, LTFU & 114 & $26.1 \%$ \\
IPT administered & \\
Completed IPT & 322 & $73.9 \%$ \\
Discontinued by patient & 284 & $88.2 \%$ \\
Discontinued due to side effects & 5 & $1.6 \%$ \\
AST and/or ALT over 200 IU/L & 33 & $10.2 \%$ \\
Nausea and pruritis & 26 & $78.8 \%$ \\
\hline
\end{tabular}

Notes. ${ }^{\dagger}$ While IPT was prescribed to 325 patients, three patients did not wish to start treatment. TST, tuberculin skin test; LTBI, latent tuberculosis infection; IPT, isoniazid preventive therapy; LTFU, loss to follow-up; AST, aspartate transaminase; ALT, alanine aminotransferase; IU/L, international units per liter.

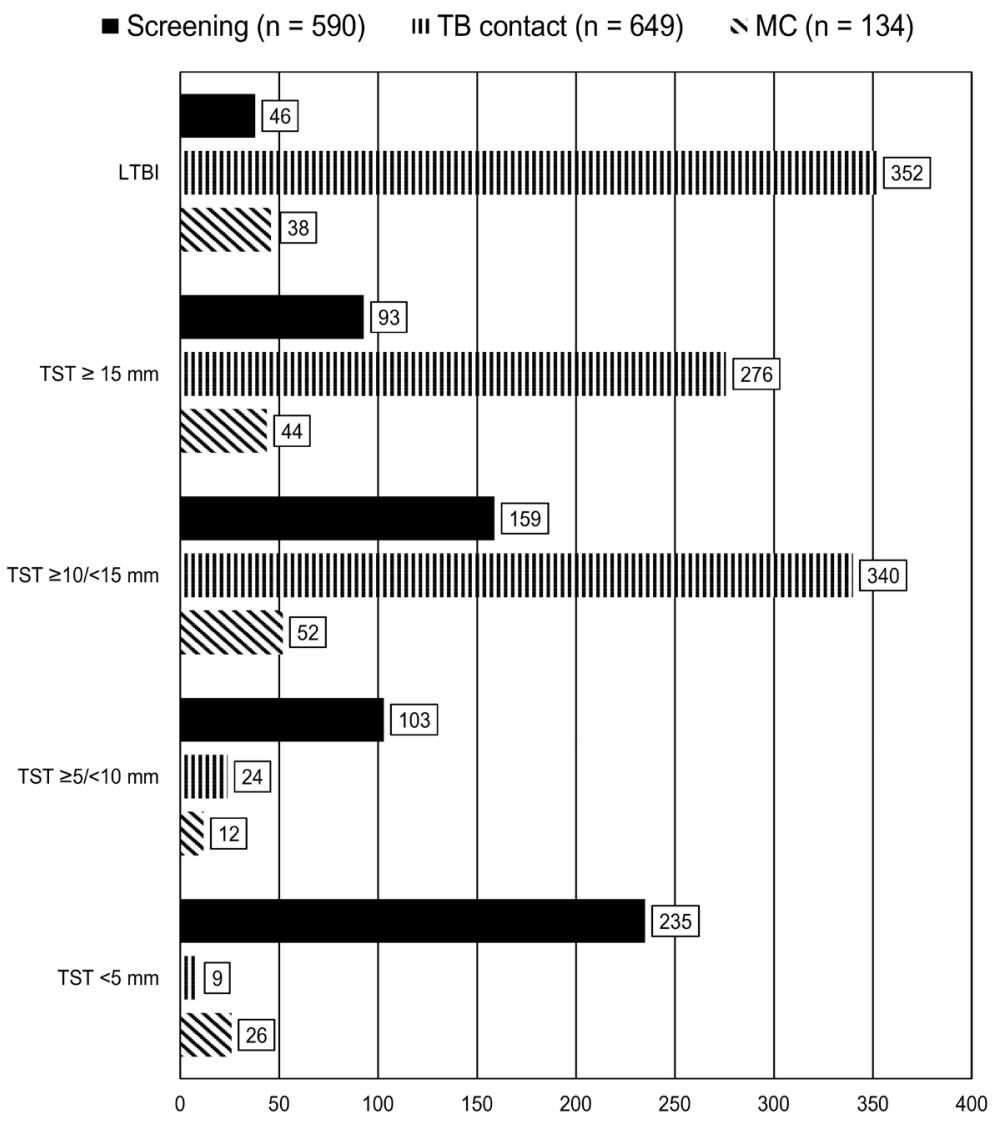

Notes. TST, tuberculin skin test; LTBI, latent tuberculosis infection.

Figure 2. Numerical TST values, reason for TST, and classification by pulmonologist. 
IPT was prescribed to $325(74.5 \%)$ of the LTBI patients, of whom $284(87.4 \%)$ completed treatment. The remaining 529 patients $(54.8 \%)$ with a positive TST result, of whom 91 were lost to follow up after the first visit to the pulmonologist, were not classified as LTBI by the pulmonologist, although $174(32.9 \%)$ had a TST result that was $15 \mathrm{~mm}$ or greater. In the latter group, 142 patients were not prescribed IPT by judgement of the pulmonologist, while the other 32 patients with a TST result of $15 \mathrm{~mm}$ or greater did not return to the pulmonologist after their first consultation.

The reason a TST was performed, the numerical value of the TST, and the classification of the TST result by the pulmonologist are shown in Figure 2. In the screening group of 590 patients ( $43 \%$ of the study cohort), a total of 46 patients (7.8\%) were classified as LTBI, but 64 patients (out of 93 with a TST result of 15 $\mathrm{mm}$ or greater) were not classified as LTBI and were assigned to a wait-and-see policy. Also, in the screening group, 159 patients had a TST result between $10 \mathrm{~mm}$ and under $15 \mathrm{~mm}$, of whom 17 (10.7\%) were classified as LTBI. In the TB contact tracing group of 649 patients ( $47.3 \%$ of the study cohort), 352 patients (54.2\%) were designated as LTBI. Even in the TB contact tracing group, 93 patients (out of 276 with a TST result of $15 \mathrm{~mm}$ or greater) were not classified as LTBI and were assigned to the wait-and-see policy. In the medical condition group of $134 \mathrm{pa}$ tients (9.8\% of the study cohort), 38 patients (28.4\%) were classified as LTBI (see Table 2).

During follow-up, three patients with a TST result between 10 and $14 \mathrm{~mm}$ developed active pulmonary TB between 3 to 7 years after their first visit to the pulmonologist. Two patients were from the TB contact tracing group and had been classified as LTBI and were prescribed IPT, which was rejected by one patient and discontinued by the other patient due to elevated liver enzymes, alanine amino transferase (ALT) and aspartate aminotransferase (AST) (over two times the upper limit on two consecutive occasions that were at least 1 week apart). The third patient was from the medical condition group and had been treated for non-TB pneumonia in 2015 and presented with pulmonary TB in 2018. After presenting with active TB disease, these three patients were successfully treated with first line tuberculostatics [18]. Regarding progression to TB, three TB cases correspond to an incidence rate of 0.7 per 1000 person-years among those with a positive TST result.

Regarding risk factors for TB, HIV was reported in 10 patients (0.7\%) with 6 patients being on antiretroviral therapy (ART), of which 5 could be prescribed IPT; 55 patients (4\%) with a positive TST result had DM, 25 were classified as LTBI, 17 completed IPT; 8 female patients had cancer $(0.6 \%), 6$ cases of breast cancer and two cases of ovary cancer of whom two were prescribed IPT; and 6 patients (0.4\%) had chronic renal failure, of whom two were prescribed IPT.

In our database, we came across three foreign patients with a TST result of 15 $\mathrm{mm}$ who had been BCG vaccinated in their home country. An additional 14 patients were noted with nontuberculous mycobacteria (NTM) in their sputum, with their TST result ranging from $0 \mathrm{~mm}$ to $21 \mathrm{~mm}$. 
Table 2. Patient classification and the reason for TST.

\begin{tabular}{|c|c|c|}
\hline Classification & $\mathbf{n}$ & $\%$ \\
\hline Total & 1373 & $100 \%$ \\
\hline Screening group ${ }^{\dagger}$ & 590 & $43 \%$ \\
\hline TST negative & 337 & $57.1 \%$ \\
\hline TST, positive: No LTBI & 207 & $35.1 \%$ \\
\hline TST under $15 \mathrm{~mm}$ & 143 & $69.1 \%$ \\
\hline$T S T \geq 15 \mathrm{~mm}$ & 64 & $30.9 \%$ \\
\hline TST, positive: LTBI & 46 & $7.8 \%$ \\
\hline IPT completed & 31 & $67.4 \%$ \\
\hline Other (LTFU, IPT discontinued, etc.) & 15 & $32.6 \%$ \\
\hline Contact tracing group ${ }^{\dagger}$ & 649 & $47.3 \%$ \\
\hline TST negative & 33 & $5.1 \%$ \\
\hline TST, positive: No LTBI & 264 & $40.7 \%$ \\
\hline TST under $15 \mathrm{~mm}$ & 171 & $64.8 \%$ \\
\hline$T S T \geq 15 \mathrm{~mm}$ & 93 & $35.2 \%$ \\
\hline TST, positive: LTBI & 352 & $54.2 \%$ \\
\hline IPT completed & 228 & $64.8 \%$ \\
\hline Other (LTFU, IPT discontinued, etc.) & 124 & $35.2 \%$ \\
\hline Medical condition group ${ }^{\dagger}$ & 134 & $9.8 \%$ \\
\hline TST negative & 38 & $28.4 \%$ \\
\hline TST, positive: No LTBI & 58 & $43.3 \%$ \\
\hline TST under $15 \mathrm{~mm}$ & 41 & $70.7 \%$ \\
\hline$T S T \geq 15 \mathrm{~mm}$ & 17 & $29.3 \%$ \\
\hline TST, positive: LTBI & 38 & $28.4 \%$ \\
\hline IPT completed & 25 & $65.8 \%$ \\
\hline Other (LTFU, IPT discontinued, etc.) & 11 & $28.9 \%$ \\
\hline
\end{tabular}

Notes. ${ }^{\dagger} \mathrm{A}$ chi-square test of independence was used to compare the proportion LTBI to that of non-LTBI cases within each TST group $-\chi^{2}(2, \mathrm{~N}=965)=308.3, p<.001$. TST, tuberculin skin test; LTBI, latent tuberculosis infection; IPT, isoniazid preventive therapy; LTFU, loss to follow-up; AST, aspartate transaminase; ALT, alanine aminotransferase; IU/L, international units per liter

Our cohort also had 11 children under the age of 5, of whom $6(55 \%)$ had a negative TST result and thus no follow-up. Of the remaining 5 children with a positive TST result, four had no follow-up or were lost to follow-up, including one child (9\%) classified as LTBI. One child with a positive TST result was assigned to a wait-and-see policy and completed 2 years of observation without displaying signs of active TB.

\section{Discussion and Conclusion}

In our study cohort, 4 out of 10 patients had a TST done for screening purposes, 
of whom less than 8 out of 100 were ultimately classified as LTBI. Regarding the patients with a positive TST result, more than half were not classified as LTBI according to the ATS guidelines.

While the ATS recommends a TST cut-off value of $5 \mathrm{~mm}$ for recent contacts of TB case patients (9), and 24 patients in our cohort did meet this criterion, their TST results between $5 \mathrm{~mm}$ and under $10 \mathrm{~mm}$ were designated TST negative and not classified as LTBI by pulmonologists because their anamnesis yielded no indication of overt exposure to TB and because of the abundant prevalence of NTM [16] in tropical Suriname. None of these patients developed active TB during the following 1 to 9 years (until the end of 2020), but latent TB infection is still possible [5]. On the other hand, these positive TST results may have been the result of cross-reactions with NTM [19]. Incorrect administration of PPD or TST reading errors could also play a role, but this seems less likely. A positive TST result due to BCG vaccinations may be ruled out because mass BCG vaccinations in Suriname were suspended in 1956.

In our opinion, the high proportion of positive TST results combined with the relative paucity of progression to active TB cases during follow-up-especially among those in which, based on clinical judgment, it was decided not to prescribe IPT-is due to the arbitrarily opted cut-off value of $10 \mathrm{~mm}$ for the TST. In all three indication groups, there were patients with a TST result of over $15 \mathrm{~mm}$ that were not designated as LTBI but were submitted to the wait-and-see policy. The reason for this approach could not be clarified. However, none of these patients developed active TB. The three patients with a positive TST result who did progress to active TB, years after initial presentation, could be cases of reinfection and not necessarily reactivation [20]. Still a more comprehensive policy in the approach of these patients is warranted and therefore included in the general approach that we propose below.

The TST remains a valuable tool in the diagnosis of LTBI, especially in resource-limited settings and in the absence of advanced diagnostic means. We observed that there are still indications for performing TSTs in Suriname that are not supported by ATS or other guidelines. The requirement of a TST before being allowed to emigrate to another country is not subject to our decision making, and therefore cannot be abandoned, but the cut-off value for the designation of a positive result should be raised to $15 \mathrm{~mm}$ in people with no TB risk factors. Given the low TB incidence, requesting a TST for elderly home admissions or work permits is not indicated and should in our view be abandoned. In such cases anamnesis, physical examination, and chest $\mathrm{X}$-rays should suffice to rule out infectious tuberculosis.

The cut-off value of $5 \mathrm{~mm}$ for people with HIV should be maintained and followed by an examination of sputum using the GeneXpert test. If this test is negative, initiation or continuation of ART should be advised. This advice is based on our finding that most HIV infected TB patients in Suriname have low CD4 counts and/or are not using ART [21]. 
The cut-off value of $5 \mathrm{~mm}$ should also be used and designated positive for those in recent contact with TB patients. For patients with medical conditions the cut-off value should be related to TB exposure and illness being a risk factor for TB, as advocated by the ATS [9].

All patients with a TST result of over $15 \mathrm{~mm}$ should be considered infected, but before the diagnosis of LTBI is considered and IPT is offered, it is important to verify that such a patient does not have active TB. If IPT is contra-indicated, rejected, or terminated due to side effects, patients should be followed by the pulmonologist and properly instructed to report symptoms that could indicate active TB.

Regarding children, the very low number of children under 5 years of age with a positive TST result in our cohort is probably because children are preferentially referred to the pediatrician and does not allow for any conclusions or suggestions about alterations to the algorithm applied to this age group.

In view of our results, we recommend the TST cut-off value to be set at $15 \mathrm{~mm}$ for people with no risk factors for TB, based on the following data: Suriname is a low TB incidence country, with an abundant prevalence of NTM and historical nonspecific tuberculin reactivity, where progression to active $\mathrm{TB}$-in persons with a TST test designated positive by the current NTP guidelines-is very low.

The strength of our study is the sample size of the population that could be evaluated during a prolonged testing period. We assume that our study cohort is representative for the tested population in Suriname since people with a positive TST result are almost exclusively referred to the pulmonologist. A limitation of this study is that while our outpatient clinic is the only pulmonary medicine clinic in Suriname, we may have missed patients with a positive TST result who did not visit our clinic after referral by the NTP, particularly people living in remote areas of the country with limited access to health care. Another limitation of our study concerns patient follow-up. Over half of patients with a positive TST result, including one in four patients classified as LTBI, were lost to follow-up or had no follow-up appointment with the pulmonologist. We may have missed people developing active TB who moved abroad and people who have died of TB without being diagnosed as such.

In conclusion, considering the incidence of TB and NTM in Suriname, our study emphasizes that TST results should be interpreted depending on the reason the TST was requested-in line with TB risk assessments and in conjunction with the incidence of TB and NTM - as advocated by the ATS. Diagnosis of LTBI should be made considering the TST in relation to regional TB incidence, patient exposure to TB and chest X-ray abnormalities compatible with undiagnosed TB. Adherence to this policy may lead to healthcare cost reductions and could result in less anxiety and less inconvenience for patients. In our opinion the TST remains a valuable tool in the diagnosis of LTBI, especially in resource-limited settings and in the absence of advanced diagnostic means. As such, our results probably may be of value to communities with a comparable TB incidence. 


\section{Acknowledgements}

We would like to thank P. Herkul and M. Simson, fellow pulmonologists from the pulmonary medicine clinic of the Academic Hospital of Paramaribo, for sharing relevant data.

\section{Authors' Contributions}

F. Gopie: Conceptualization, Data Curation, Investigation, Writing-Original Draft. A. Hassankhan: Data Curation, Formal Analysis, Writing-Review \& Editing. C.W.R. Zijlmans: Conceptualization, Supervision, Writing-Review \& Editing S. Vreden: Conceptualization, Supervision, Writing-Review \& Editing

\section{Conflicts of Interest}

The authors declare no conflicts of interest regarding the publication of this paper.

\section{References}

[1] Small, P.M. and Fujiwara, P.I. (2001) Management of Tuberculosis in the United States. New England Journal of Medicine, 345, 189-200. https://doi.org/10.1056/NEJM200107193450307

[2] Arroyo-Ornelas, M.A., Arenas-Arrocena, M.C., Estrada, H.V., Castaño, V.M. and López-Marín, L.M. (2012) Immune Diagnosis of Tuberculosis through Novel Technologies. In: Cardona, P.J., Ed., Understanding Tuberculosis. Global Experiences and Innovative Approaches to the Diagnosis, IntechOpen, London, 379-394.

[3] Cohen, A., Mathiasen, V.D., Schön, T. and Wejse, C. (2019) The Global Prevalence of Latent Tuberculosis: A Systematic Review and Meta-Analysis. European Respiratory Journal, 54, Article ID: 1900655. https://doi.org/10.1183/13993003.00655-2019

[4] Houben, R.M. and Dodd, P.J. (2016) The Global Burden of Latent Tuberculosis Infection: A Re-Estimation Using Mathematical Modelling. PLoS Medicine, 13, Article ID: e1002152. https://doi.org/10.1371/journal.pmed.1002152

[5] Kiazyk, S. and Ball, T.B. (2017) Latent Tuberculosis Infection: An Overview. Canada Communicable Disease Report, 43, 62-66. https://doi.org/10.14745/ccdr.v43i34a01

[6] Shea, K.M., Kammerer, J.S., Winston, C.A., Navin, T.R. and Horsburgh Jr., C.R. (2014) Estimated Rate of Reactivation of Latent Tuberculosis Infection in the United States, Overall and by Population Subgroup. American Journal of Epidemiology, 179, 216-225. https://doi.org/10.1093/aje/kwt246

[7] World Health Organization (2018) Latent TB Infection: Updated and Consolidated Guidelines for Programmatic Management. WHO/CDS/TB/2018.4. World Health Organization, Geneva.

[8] Zhou, G., Luo, Q., Luo, S., Teng, Z., Ji, Z., Yang, J., Wang, F., Wen, S., Ding, Z., Li, L. and Chen, T. (2020) Interferon- $\gamma$ Release Assays or Tuberculin Skin Test for Detection and Management of Latent Tuberculosis Infection: A Systematic Review and Meta-Analysis. The Lancet Infectious Diseases, 20, 1457-1469. https://doi.org/10.1016/S1473-3099(20)30276-0

[9] American Thoracic Society (2000) Targeted Tuberculin Testing and Treatment of Latent Tuberculosis Infection. American Journal of Respiratory and Critical Care Medi- 
cine, 161, S221-S247. https://doi.org/10.1164/ajrccm.161.supplement 3.ats600

[10] Rose, D.N., Schechter, C.B. and Adler, J.J. (1995) Interpretation of the Tuberculin Skin Test. Journal of General Internal Medicine, 10, 635-642. https://doi.org/10.1007/BF02602749

[11] Menzies, R.I. and Tuberculin Skin Testing (2000) In: Reichman, L.B. and Hershfield, E.S., Eds., Tuberculosis. A Comprehensive International Approach, Second Edition, Revised and Expanded, Marcel Dekker, New York, 279-322.

[12] World Health Organization (2019) Global Tuberculosis Report 2019. WHO/CDS/ TB/2019.15. World Health Organization, Geneva.

https://www.who.int/publications/i/item/global-tuberculosis-report-2019

[13] National Tuberculosis Program, Bureau of Public Health, Ministry of Health (2012) Nationale Richtlijnen voor Tuberculose. Bureau of Public Health, Ministry of Health, Suriname.

[14] (2002) Suriname Medical Bulletin Dec. 2002. Vol. XVII nr. 4-2002.

[15] Menke, J. (Ed.) (2016) Mozaïek van het Surinaamse Volk: Volkstellingen in Demografisch, Economisch en Sociaal Perspectief. 5th Edition, Algemeen Bureau voor de Statistiek/Anton de Kom Universiteit van Suriname, Institute for Graduate Studies and Research, Suriname.

[16] Gopie, F.A., Hassankhan, A., Zijlmans, W., de Lange, W.C.M., Vreden, S.G.S. and van Ingen J. (2020) Nontuberculous mycobacteria Species in Sputum Cultures from Suriname. International Journal of Tuberculosis and Lung Disease, 24, 1106-1108. https://doi.org/10.5588/ijtld.20.0249

[17] Ohshimo, S., Guzman, J., Costabel, U. and Bonella, F. (2017) Differential Diagnosis of Granulomatous Lung Disease: Clues and Pitfalls: Number 4 in the Series "Pathology for the Clinician" Edited by Peter Dorfmüller and Alberto Cavazza. European Respiratory Review, 26, Article ID: 170012. https://doi.org/10.1183/16000617.0012-2017

[18] World Health Organization (2010) Treatment of Tuberculosis: Guidelines. 4th Edition, WHO/HTM/TB/2009.420. World Health Organization, Geneva.

[19] Bahrmand, A.R., Samar, H.M., Khalilzadeh, L., Bakayev, V.V., Yaghli, M. and Babaei, M.H. (1996) Detection and Identification of Non-Tuberculous mycobacterial Infections in 6,472 Tuberculosis Suspected Patients. Scandinavian Journal of Infectious Diseases, 28, 275-278. https://doi.org/10.3109/00365549609027172

[20] Cardona, P.J. (2016) Reactivation or Reinfection in Adult Tuberculosis: Is That the Question? International Journal of Mycobacteriology, 5, 400-407. https://doi.org/10.1016/j.ijmyco.2016.09.017

[21] Gopie, F.A., Hassankhan, A., Ottevanger, S., Krishnadath, I., de Lange, W., Zijlmans, C.W.R. and Vreden, S. (2021) Ethnic Disparities in Tuberculosis Incidence and Related Factors among Indigenous and Other Communities in Ethnically Diverse Suriname. Journal of Clinical Tuberculosis and Other Mycobacterial Diseases, 23, Article ID: 100227. https://doi.org/10.1016/j.jctube.2021.100227 